\title{
BMJ Open Effect of exercise-based management on multidirectional instability of the glenohumeral joint: a pilot randomised controlled trial protocol
}

\author{
Sarah A Warby, ${ }^{1,2}$ Jon J Ford, ${ }^{1}$ Andrew J Hahne, ${ }^{1}$ Lyn Watson, ${ }^{1,2}$ Simon Balster, ${ }^{2,3}$ \\ Ross Lenssen, ${ }^{1,2,3}$ Tania Pizzari ${ }^{1}$
}

To cite: Warby SA, Ford JJ, Hahne AJ, et al. Effect of exercise-based management on multidirectional instability of the glenohumeral joint: a pilot randomised controlled trial protocol. BMJ Open 2016;6:e013083. doi:10.1136/bmjopen-2016013083

- Prepublication history and additional material is available. To view please visit the journal (http://dx.doi.org/ 10.1136/bmjopen-2016013083).

Received 17 June 2016 Accepted 26 July 2016

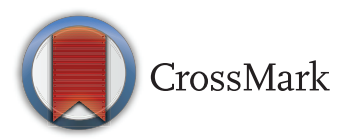

\footnotetext{
${ }^{1}$ Department of

Rehabilitation, Nutrition and Sport, La Trobe University, Bundoora, Victoria, Australia ${ }^{2}$ LifeCare Prahran Sports Medicine Centre, Prahran, Victoria, Australia

${ }^{3}$ Melbourne Orthopedic Group, Melbourne, Victoria, Australia
}

Correspondence to Sarah A Warby; SWarby@latrobe.edu.au

\begin{abstract}
Introduction: The most commonly recommended treatment for multidirectional instability (MDI) of the shoulder is exercise. Despite this recommendation, there is limited evidence to support the effectiveness of exercise. The aim of this paper is to describe a pilot randomised controlled trial comparing the effectiveness of 2 exercise programmes on outcomes of participants with MDI.
\end{abstract}

Methods and analysis: Consenting participants between 12 and 35 years, with non-traumatic MDI will be randomly allocated to participate in either the Rockwood Instability programme or the Watson MDI programme. Both programmes involve 1 consultation per week for 12 weeks with a physiotherapist to prescribe and progress a home exercise programme. Outcomes will be assessed at baseline, 6, 12, 24 and 52 weeks. Primary outcome measures include the Melbourne Instability Shoulder Score and Western Ontario Shoulder Index. Secondary outcomes include scapular coordinates, scapular upward rotation angles, muscle strength, symptomatic onset, limiting factor and angle of limiting factor in abduction range, incidence of complete glenohumeral joint dislocation, global rating of change, satisfaction scores, the Orebro Musculoskeletal Pain Questionnaire, adverse events and compliance with the home exercise programme. Data will be analysed on intention-to-treat principles and a per protocol basis.

Discussion: This trial will evaluate whether there are differences in outcomes between the Rockwood and the Watson MDI programmes for participants with MDI.

Ethics and dissemination: Participant confidentiality will be maintained with publication of results. Ethics approval: Faculty of Health Sciences (FHEC12/201). Trial registration number: ACTRN12613001240730; Pre-results.

\section{INTRODUCTION}

Multidirectional instability (MDI) is symptomatic glenohumeral joint subluxation or dislocation occurring in more than one

\section{Strengths and limitations of this study}

- To the authors' knowledge this is the first pilot randomised controlled trial (RCT) comparing the effect of two rehabilitation programmes for patients with multidirectional instability (MDI), and the first RCT to test this patient population using instability-specific outcomes. Findings are likely to be clinically relevant and useful for physiotherapists treating MDI conservatively.

- Our strict inclusion criteria for MDI participants will increase the likelihood of a more homogeneous MDI group and reduce selection bias.

- Blinding of participants, blinding of assessors of outcomes, detailed standardisation of treatment protocols and a rigorous physiotherapy training and mentoring programme all enhance internal validity of this trial.

- Owing to PhD time limits of the primary researcher and the lack of available data on primary outcome measures to calculate more accurate sample sizes, this study will not be able to reach the sample size required for a definitive RCT. Therefore, it will be a pilot trial to determine the feasibility of a full powered RCT and allow more accurate sample size calculations.

- Physiotherapists remain unblinded to the intervention they are delivering which could introduce therapist bias.

direction. ${ }^{1-3}$ There is general agreement that the aetiology of this condition is due to repetitive microtrauma imposed on a congenitally lax and redundant joint capsule. ${ }^{3-7}$ People with MDI typically have reduced scapular upward rotation, an imbalance of muscle strength and suboptimal neuromuscular control of shoulder function when compared with normal controls. ${ }^{3} 68$ People with MDI can present with a variety of symptoms ranging from reports of vague shoulder pain to daily occurrences of symptomatic subluxations and dislocations with activities of daily 
living. ${ }^{1}$ Depending on the severity of the condition, MDI can adversely affect quality of life due to pain, activity avoidance, occupational restrictions or reduced sporting performance. ${ }^{1}$ Shoulder instability is one of the most common shoulder pathologies, ${ }^{9}$ especially in the younger sporting population. ${ }^{10-13}$ MDI is a subset of atraumatic shoulder instability ${ }^{114}$ and although prevalence data are lacking, ${ }^{15}$ experts report that the condition is becoming more recognised in the clinical setting. ${ }^{16}$

The most commonly recommended treatment for MDI is exercise-based management. ${ }^{1-4} \quad 6 \quad 17 \quad 18$ This approach is based on the mechanism of strengthening the scapular and rotator cuff muscles, compensating for the lack of passive stability and thereby assisting in active control of the shoulder. ${ }^{2}{ }^{3}{ }^{6}$ Recent systematic reviews have found some evidence supporting the effectiveness of exercise for MDI; however, the quality of evidence was very low. ${ }^{19}{ }^{20}$ Issues identified in these reviews included a high level of bias across the included studies, heterogeneous patient samples, poorly defined exercise protocols and a lack of baseline outcome measures. On the basis of this literature, therapists have very low-quality evidence on which to base their treatment choices.

To date, the Rockwood Instability programme is the only published MDI protocol that outlines enough detail for physiotherapists to replicate in the clinical setting. ${ }^{17}$ Rockwood and Burkhead reported that $87 \%$ of their MDI participants had a good-to-excellent outcome with their programme; yet, their sample size was small, outcomes measures were not specific or sensitive to measuring change in the instability population, and were only taken retrospectively. ${ }^{19}{ }^{20}$ In addition, the Rockwood programme has no specific scapular muscle retraining and lacks exercise drills in higher degrees of shoulder elevation. ${ }^{17}$

The Watson MDI programme ${ }^{21} 22$ has been developed for the conservative treatment of MDI. The programme has been shown to significantly improve scores on the Melbourne Instability Shoulder Score (MISS) and the Western Ontario Shoulder Index (WOSI) for a group of MDI participants in a pre-post trial design. ${ }^{23}$ The Watson programme focuses on re-establishing patient-specific scapular control, typically scapular upward rotation, prior to any rotator cuff or deltoid strengthening. Scapular control is emphasised throughout the programme and exercises progress into functional and sports-specific ranges.

Currently, no published RCTs have compared the effect of one standardised exercise programme against another, nor have functional and instability-specific outcomes been used to measure change in this population. The aim of this paper is to describe the design of a pilot randomised controlled trial (RCT) comparing the Rockwood Instability programme with the Watson MDI programme for people with MDI. We hypothesise that the Watson MDI programme will produce clinically and statistically better outcomes over the Rockwood programme at the primary 12-week time point, due to its focus on achieving and maintaining scapular control and progressing exercises into functional ranges.
This trial may assist in establishing guidelines for exercise prescription, improve outcomes for people with MDI and lay foundations for larger RCTs to evaluate exercise for shoulder instability.

\section{METHODS AND ANALYSES}

Reporting of this protocol will adhere to the Standard Protocol Items for Randomised Trials (SPIRIT) ${ }^{24}$ and CONSORT $^{25}$ statements.

\section{Study aims}

The primary aim of this trial is to compare the relative effectiveness of the Rockwood Instability programme and the Watson MDI programme on functional and instability-specific outcomes, scapular measures and muscle strength of participants with non-traumatic MDI.

\section{Study design}

This will be a multicentre pilot RCT. An overview of the trial procedure is outlined in figure 1. Participants will be randomly allocated to one of two 12-week exercise programmes, the Rockwood Instability programme or the Watson MDI programme. The fundamentals of both programmes have evidence of beneficial effects on people with MDI, ${ }^{7} 172326$ thus clinical equipoise is maintained. ${ }^{27}$ Outcome measures will be taken at baseline, $6,12,24$ and 52 weeks postrandomisation. The primary comparison for this study will be at the 12-week time point.

After the 12-week outcome measures have been obtained, participants who score less than the minimal clinically important difference (MCID) on both primary outcome measures ( 5 points on the MISS and $10.4 \%$ on the WOSI) will have the option to swap to the other exercise programme for a subsequent 12 weeks. The feasibility of this study would be limited without an optional cross-over design as referrers expressed a reluctance to refer patients with this complex pathology into the trial without the option of receiving the alternative treatment programme, should they score less than the MCID on both primary outcomes. The authors acknowledge that this study design is not a true cross-over design due to the second phase of treatment being optional and the absence of a washout period. ${ }^{28} \mathrm{~A}$ true washout period is almost impossible to implement in any exercise-based study due to the central effect of motor learning; ${ }^{29}{ }^{30}$ however, as the threshold for crossover is to score less than the MCID on both primary outcome measures, it can be considered that the participant's functional measures are close to baseline ${ }^{31}$ and the exercise programme was of little benefit. Owing to the cross-over, the 12-week follow-up will be the primary outcome point. The 24-week and 52-week follow-ups will be secondary outcome points of this study. Confounding due to any carry over effect will be minimised by detailed reporting of the cross-over procedure. ${ }^{28}$ 


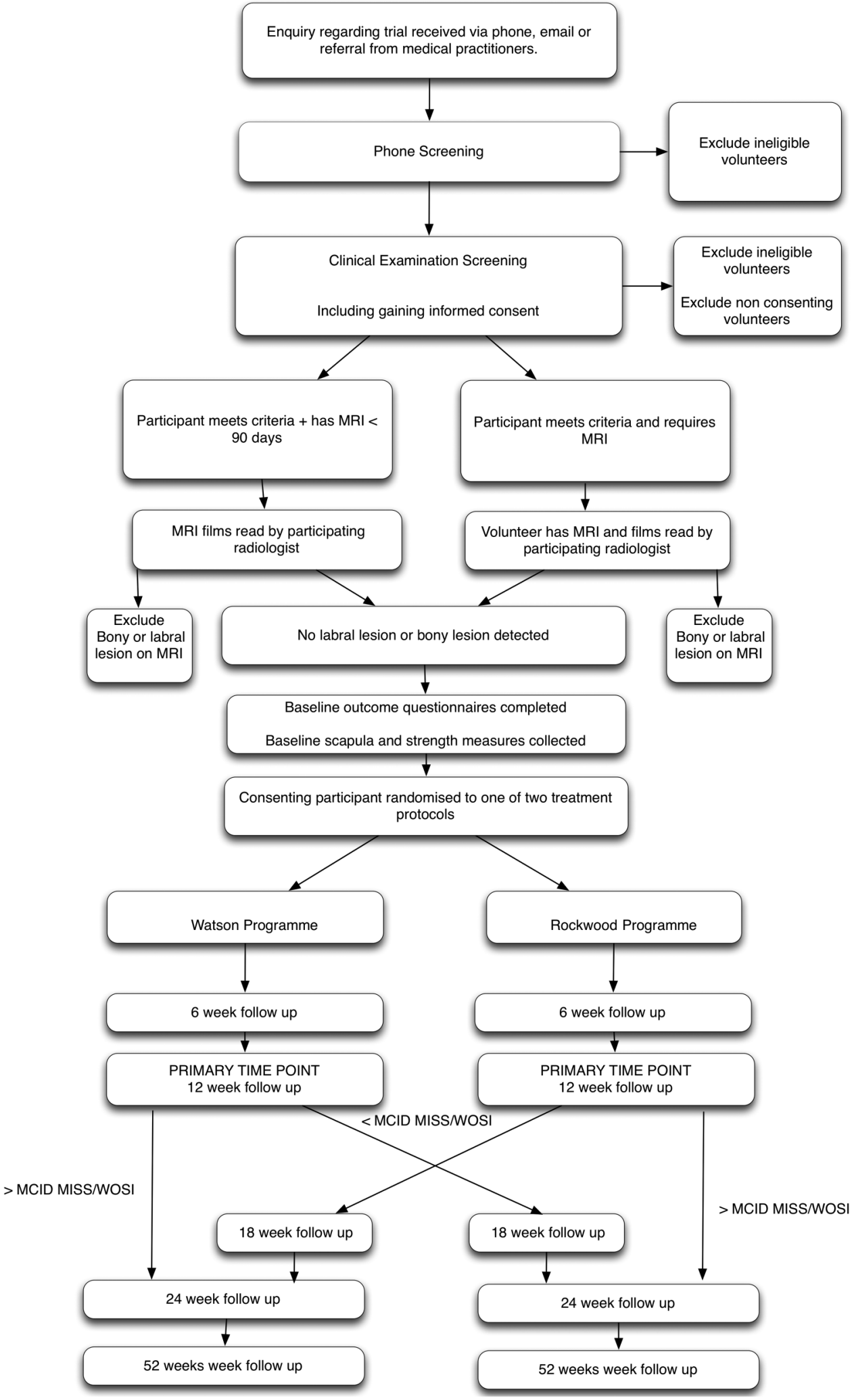

Figure 1 Overview of trial procedure. MCID, minimal clinically important difference; MISS, Melbourne Instability Shoulder Score; WOSI, Western Ontario Shoulder Index.

\section{Sample size}

The sample size required for a definitive RCT would be a total of 328 participants (164 in each group) to detect a MCID of 5 points on the MISS outcome measure, assuming a SD of 16 ( $\alpha$ of 0.05 and a power of $80 \%) .{ }^{32}{ }^{33}$ Only one pre-post study has reported the SD for the MISS ${ }^{32}$ and none are reported for the WOSI. Therefore, the proposed study will be a pilot study to 
determine the feasibility of a fully powered RCT, and to clarify the sample size calculation. Recruitment for this study started in January 2014.

\section{Setting and recruitment}

The treatments will be conducted at one of seven private physiotherapy practices that are part of the LifeCare Health network throughout metropolitan Melbourne, Australia. Participants will be sought via referrals from orthopaedic surgeons, sports physicians and physiotherapists. Potential referrers will be informed of the trial and the referral process via formal meetings, personal correspondence, department lectures and trial information sheets.

\section{Eligibility and screening}

Participants

Participants included in this trial will be between the ages of 12 and 35 years ${ }^{34}$ with non-traumatic, symptomatic shoulder instability in at least two directions ${ }^{13}$ and no labral or bony lesions detected on MRI. Table 1 summarises the inclusion and exclusion criteria.

\section{Phone screening}

Potential participants will initially undergo a preliminary screening for eligibility via telephone. The purpose of the phone screening is to eliminate participants who are clearly ineligible. In particular, the assessor will carefully question potential participants to determine if any significant history of trauma to the shoulder exists. Participants will be excluded if they report any significant history of trauma to the affected shoulder as participants with a significant history of trauma are more likely to have a structural lesion and predominantly unidirectional pathology. ${ }^{14} 35$ A significant history trauma was defined as contact with an external object (such as a fall, impact with another body or surface) with lock out of the glenohumeral joint and conscious awareness by the participant of a sudden onset of pain. ${ }^{18}$

\section{Clinical examination screening}

Participants found to be potentially eligible after the phone screening will be invited to attend a subjective and physical examination by one of the three experienced shoulder physiotherapists. Table 2 outlines the components of the clinical examination. Additional details and rationale for each clinical examination component are outlined in the online supplementary appendix 1. For the purposes of this trial, MDI will be defined as symptomatic instability in at least two directions. ${ }^{8} 5253$ To be positive for instability, the participant must have apprehension (which may include muscles spasm or guarding) on testing and not just pain or signs of laxity. ${ }^{38} 4454$

\section{Signing of consent forms}

On meeting all of the inclusion criteria in the clinical examination, participants will again be informed of the nature of the trial and asked to sign a consent form. In addition to signing their own consent form, participants under the age of 18 years will need to have their parent or guardian sign a separate consent form to participate in the study (see online supplementary appendix 2 ). At the time of signing the consent form, potential participants will be informed that inclusion in the trial is on the provision that their MRI meets the inclusion criteria.

\section{MRI}

On meeting the assessment criteria in the clinical examination screening, the potential participant will be invited to have an MRI to investigate the presence of any structural lesion of the affected shoulder. Participants with a bony lesion (Hill Sachs or bony Bankart) or labral lesion (SLAP, labral Bankart) will be excluded from the trial as they are more likely to have a history of major shoulder trauma, and predominantly unidirectional pathology, ${ }^{14} 18$ and better outcomes with surgical stabilisation. ${ }^{6} 141718$ All MRI films will be read and reported on by a senior radiologist. Participants will be informed of their MRI results by the primary researcher (SAW) by phone, and if eligible, have their first treatment session arranged at their closest participating treatment centre.

\section{Randomisation and allocation}

Eligible participants who provide written consent will be randomised into the Rockwood Instability programme or the Watson MDI programme. To ensure allocation concealment, an offsite randomisation schedule will be used. The randomisation schedule will be prepared in advance by a researcher at La Trobe University (AJH) who will have no contact with any participants throughout the trial and will not be involved in the recruitment, screening, assessment, enrolment or treatment process. The randomisation sequence (with random block sizes) will be generated using a web-based randomisation programme (http://www.randomisation.com) with the sequence transferred onto a computer spreadsheet. Randomisation will be stratified for the treating practitioner. Allocation of participants in accordance with the randomisation schedule will be undertaken by the same researcher at La Trobe University (AJH) who will be the only person with access to the allocation spreadsheet during the trial. To enrol a participant, the primary researcher (SAW) will email the consenting participant's name, date of birth and treating physiotherapist to the La Trobe University researcher (AJH). These details will be entered into the allocation spreadsheet and the next treatment allocation and participant identification number with be emailed directly to the treating physiotherapist.

\section{Treating physiotherapists and treatment fidelity}

Treating physiotherapists will have worked in private practice for at least 2 years and be engaged with an ongoing clinical mentoring programme. An additional 
Table 1 Inclusion and exclusion criteria

Inclusion criteria
Initial phone screening
Reports of shoulder region discomfort, pain or apprehension/guarding with
movement
Willingness to participate in a 12-week exercise programme
Age between 12 and 35 years inclusive

$M R I$

Normal MRI

Normal anatomical variant accepted: labral deficiency, labral recess, glenoid dysplasia

Minor pathologies accepted: bursitis, small rotator cuff tears, labral 'fraying'

\section{Clinical examination screening}

Clinically diagnosed MDI, with symptomatic instability in at least 2 directions. The diagnosis of MDI must be defined by apprehension or guarding with the following tests:

A positiveł sulcus sign

AND

For one direction, at least 2 out of 3 positive for the following tests:

- Draw test adducted

- Draw test abducted

- Apprehension test

The 'effect of manual correction on scapula biomechanics' MUST symptomatically improve a participant's abduction range of motion by a minimum of $20^{\circ}$, significantly reduce a participant's pain or guarding in abduction, or improve a participant's strength on an isometric test ${ }^{21}$ (table 2 and online supplementary appendix 1).

\section{Exclusion criteria}

History of significant trauma*

History of glenohumeral dislocation that requires relocation $\dagger$

Prior surgical history of the affected shoulder(s)

Unable to understand and follow instructions in English

Bony lesion (bony Bankart, Hill Sach's) or fracture

Labral lesions (SLAP, labral Bankart)

Full thickness rotator cuff tears

Full thickness bicep tear

Frank labral tears

Contraindications to MRI (eg, pacemaker, claustrophobia, pregnancy)

Non-correctable volitional instability

Non-compliance

Neurological motor deficit

Instability due to UMN or LMN lesion

Ehlers-Danlos syndrome/Marfan's syndrome

Shoulder pain that is predominantly due to cervical dysfunction including:

Cervical spine somatic referred pain

Cervical spine radicular pain

Cervical spine radiculopathy TOS

*Significant trauma defined as contact with an external object (such as a fall, impact with another body or surface) with lock out of the glenohumeral joint and conscious awareness by the patient of a sudden onset of pain.

†Relocation defined as MUA by a health professional or force applied externally by patient or other person at the time of injury to relocate the glenohumeral joint.

$\ddagger$ To be positive for instability the participant must have apprehension (which may include muscles spasm or guarding) on testing and not just pain or signs of laxity.

LMN, lower motor neuron lesion; MUA, manipulation under anesthetic; TOS, thoracic outlet syndrome; UMN, upper motor neuron lesion.

2-day training programme will occur for all treating physiotherapists led by LW and the primary researcher (SAW) and include group discussions and portions of experimental learning as outlined by Main et $a l^{55}$ for training physiotherapists delivering interventions. For the duration of the trial, quarterly workshops will be undertaken involving all treating physiotherapists to review specific cases in the context of the treatment programmes.

Trial physiotherapists will be provided with a 364 page treatment manual outlining the trial protocol, the requirements of trial physiotherapists and the details of each treatment programme. Both programmes have been standardised via detailed flow charts, adhering to guidelines outlined by either Burkhead and Rockwood ${ }^{17}$ or Watson $e t a l^{21} 22$ The flow charts contain guidelines for exercise prescription and progressions and algorithms for clinical decision-making. The treating physiotherapist will also be required to complete electronic clinical notes at each treatment session to document the assessment findings, clinical decision-making rationale, exercises prescribed and any adverse events attributed to the exercise programme. These measures adhere to the recommended requirements for ensuring treatment fidelity. ${ }^{55}$

To evaluate treatment integrity, the clinical notes for both programmes will be reviewed at 3, 6 and 12 weeks to ensure that all documentation is standardised, legible and complete. The review of clinical notes will be carried out by three of the researchers (JJF, TP, RL), who are not blinded to the treatment allocation of participants.

\section{Treatment programmes}

Two different 12-week exercise programmes will be compared (table 3). All participants will attend 1 session of 
Table 2 Clinical examination screening

\section{Clinical examination components}

History of presenting condition

The presence of volitional instability

A full history of the patient's condition will be recorded, including frequency, aetiology, direction and severity of subluxations. ${ }^{36}$

Patients who present or report a predominance of volitional instability (voluntarily sublux their glenohumeral joint on a regular basis) will be excluded, as treatment for this type of instability primarily focuses on counselling to cease the habitual subluxation. ${ }^{14}$

Diagnosis of MDI with instability tests Diagnosis must be based on:

1. A positive sulcus sign. ${ }^{37}$ The sulcus test is a valid and reliable test for inferior laxity ${ }^{38}$ with a fair to good interexaminer reliability $(60-85 \%){ }^{38-40}$ AND

2. For at least one direction (anterior and/or posterior), a positive result for at least 2 out of 3 following tests:

- Anterior and posterior draw tests $\left(10-30^{\circ} \text { abduction }\right)^{37} 41$

- Anterior and posterior draw tests in (80-120 abduction) $)^{37} 41$

- Anterior ${ }^{38} 4243$ and posterior apprehension tests ${ }^{44} 45$

Effect of manual correction on scapula biomechanics

Upward rotation test

Generalised ligament laxity

Cervical spine examination
The 'effect of manual correction on scapula biomechanics' is defined as the effect that therapist-assisted manual assistance of the scapula has on an objective test. ${ }^{21} 4647$ The effect of manual correction of the scapula will be assessed via active abduction, active flexion and isometric external rotation. In order for participants to be eligible, the effect of manual correction of the scapula must symptomatically improve abduction range by a minimum of $20^{\circ}$, significantly reduce pain or guarding in range or improve strength on an isometric test. Poor scapular positioning through range and altered muscle patterning are predominant characteristics of non-traumatic MDI. ${ }^{314} \mathrm{An}$ immediate improvement with manual assistance is likely to confirm the presence of these characteristics and indicate that the participant is appropriate for treatment with exercise.

To be eligible, participants must be able to perform a minimum of 5 repetitions of scapular upward rotation in standing position without the reproduction of any cervical spine pain or headaches. ${ }^{21}$ As both rehabilitation programmes require the participant to perform some scapular strengthening, inability to perform this test indicates that they are not appropriate for either exercise programme and may have a predominant cervical spine component to their pathology.

The Beighton Scale ${ }^{48}$ will be used to assess generalised ligament laxity and has a good to excellent reliability. ${ }^{49}$

Potential participants with a positive Spurlings test for cervical radiculopathy and radicular pain with be excluded. ${ }^{50} 51$ The test has a high sensitivity $(92 \%)^{51}$ and specificity $(93-95 \%)^{50}{ }^{51}$ for cervical radiculopathy.

MDI, multidirectional instability.

$60 \mathrm{~min}$ and 11 sessions of $30 \mathrm{~min}$ each where they will be assessed/reassessed and prescribed/progressed with a specific set of exercises. Participants in the both groups will be instructed to perform their exercises at home and/or in their gym. Equipment for the relevant programme will be supplied. Resistance bands (Theraband) for both groups will be cut to a length of $1.5 \mathrm{~m}$. Each participant will be provided with an exercise logbook, in which the participant will record the date of every home session. The treating physiotherapist will fill out these log books with the relevant programme exercise parameters at each session.

\section{The Rockwood programme}

The Rockwood programme focuses on concurrently strengthening all three parts of the deltoid, the internal and external rotators of the glenohumeral joint in two phases. Phase 1 involves five exercises for the rotator cuff and deltoid using a set of six rubber Therabands with varying resistances of $0.5,1.0,1.5,2.0,2.5$ and $3 \mathrm{~kg}$. The second phase of strengthening begins when the participant has progressed through all the resistance bands. The participant is then instructed to perform the same exercises as of phase 1 with a $4 \mathrm{~kg}$ weight using a pulley kit. The weight is then progressed in increments of $1 \mathrm{~kg}$. Therabands or weight resistance is progressed once the participant reports that the current resistance is 'relatively easy'. ${ }^{17}$ All exercises must be pain free to perform.

\section{Watson MDI programme}

The Watson programme is primarily based around maintaining good scapular control through all stages of the programme. $^{21}{ }^{22}$ Most stages have a scapula phase that the participant has to master before moving on to the arc of motion phase. Stage 1 is the foundation phase 
Table 3 Treatment programmes

\section{Rockwood programme}

Focus: concurrent rotator cuff and deltoid strengthening and push ups for scapular stability. Majority of exercises performed at $0^{\circ}$ of elevation.

Aims and exercise drills

Phase 1: aim: strength through progressive levels of Theraband resistance

Load: tan, yellow, red, green, blue Theraband

Patient standing

- ER $\left(0-45^{\circ}\right.$ ER) at $0^{\circ}$ abduction

- IR $\left(0-45^{\circ} \mathrm{IR}\right)$ at $0^{\circ}$ abduction

- Extension row to $45^{\circ}$

- Flexion

- Short lever abduction to $45^{\circ}$

- Wall, knee or full push ups (no Theraband resistance)

Phase 2: aim: strength through resistance with weights

Load: 4-5-6-7-8-9-10-11 kg with weight and pulley system Load begins at $4 \mathrm{~kg}$

Progresses in $1 \mathrm{~kg}$ increments

$9 \mathrm{~kg}$ maximum for females, $11 \mathrm{~kg}$ maximum for males

Patient standing

- ER $\left(0-45^{\circ}\right.$ ER $)$ at $0^{\circ}$ abduction

- IR $\left(0-45^{\circ} \mathrm{IR}\right)$ at $0^{\circ}$ abduction

- Extension row to $45^{\circ}$

- Flexion

- Short lever abduction to $45^{\circ}$

Wall, knee or full push ups (no Theraband resistance) Addition of shoulder shrug holding weight anteriorly with both hands

Not all exercises need to be progressed at the same time and participants may be on a different band or weight/resistance for different exercises.
Dosage

All exercises 5 repetitions with a $5 \mathrm{~s}$ hold at the end range of the exercise. All exercises are performed twice a day

\section{Watson programme}

Focus: retraining specific scapular faulty biomechanics prior to any rotator cuff/deltoid strengthening. Exercises progress into functional/sports-specific ranges.

Stage 1: scapular phase: aim: retrain scapular control Load: $0-0.5-1 \mathrm{~kg}$

- Scapular upward rotation/elevation drills in standing Arc of motion phase: aim: controlling arcs of motion (0-45 elevation)

Load: yellow-red Theraband

Patient standing

- Extension rows (from $45^{\circ}$ flexion to neutral)

- ER $\left(0-45^{\circ}\right.$ ER) at $0^{\circ}$ abduction

- IR (0-45 IR) at $0^{\circ}$ abduction

Stage 2: aim: building posterior GHJ muscle bulk

Load: green Theraband/1-2 kg

Patient standing

- Bent arm rows

- Side lying ER

- Standing Theraband rows

Stage 3: scapular phase: aim: scapular control in sagittal plane

Load: $0-0.5-1-2 \mathrm{~kg}$

- Scapular upward rotation/elevation drills in standing (sagittal plane)

Arc of motion phase: aim: sagittal plane (flexion control) in 0-90 elevation

Load: yellow-green Therabands $/ 1-3 \mathrm{~kg}$

Patient standing

- Flexion with Therabands and weights

Stage 4: scapular phase: aim: scapular control at $90^{\circ}$ of elevation

Load: red-green Therabands

- Standing row at $90^{\circ}$ of elevation

Arc of motion phase: aim: controlling arcs of motion (45-90 elevation)

Patient standing

Load: yellow-green Therabands/2-5 kg

- ER at $90^{\circ}$

- IR at $90^{\circ}$

- Flexion at $90^{\circ}$

Stage 5: aim: specific deltoid strengthening

Load: $1-4 \mathrm{~kg}+$

Patient standing

- Bent over rows

- Supine and sitting flexion

- Short lever abduction $45-60^{\circ}$

Stage 6: aim: sports-specific/functional control and strength Load: depends on participant's requirements

Drills mimic specific sporting or functional activates

Part practise to full practise

Typically start with a recruitment dosage for motor relearning $(3 \times 20,2 \times$ day $),{ }^{56}$ followed by an endurance dosage $(3 \times 10-$ $15,2 \times$ day $)$, then strength dosage in later stages $(4 \times 8-12$, every second day). ${ }^{57}$ For most exercises, repetitions are held for $3 \mathrm{~s}$. 
and focuses on retraining faulty scapular biomechanics. The clinical assessment determines the exact scapular mechanics that the patient must retrain and maintain throughout the programme, ${ }^{21}$ and most commonly incorporates scapular upward rotation. ${ }^{2}{ }^{21}$ The stages progress via an increase in load and an increase in range of glenohumeral joint elevation. The final stages incorporate functional and/or sports-specific exercises. Progression to a more difficult exercise or to the next stage of the programme is determined by the participant reaching specific exercise called 'goals'. Goals are a combination of meeting a certain number of exercise repetitions with a specified load, while maintaining adequate scapular control. The trial physiotherapist must observe the participant performing one set of each drill while maintaining adequate scapular stability (eg, maintaining scapular upward rotation and avoiding downward rotation or anterior tilt) to determine if they are ready to progress. Details of each stage and every goal of the Watson programme have been published. ${ }^{21} 22$

\section{Participant education and other co-interventions}

All participants will be educated regarding the nature of their pathology, the rationale for exercise treatment, the importance of compliance to their home programme, timeframes, goal setting and avoiding aggravating or unsafe activates. Participants will be specifically educated on the difference between pain and muscle fatigue in response to their home programme. If a participant reports a significant increase in pain during or after exercises, the trial physiotherapist can regress or alter the exercise as per programme guidelines. Manual treatment and management of glenohumeral joint inflammation are other co-interventions that may be implemented (see online supplementary appendix 3 ).

\section{Outcome measures}

The 12-week outcome point will be the primary outcome point. The majority of outcomes will be assessed via a set of self-administered questionnaires, that will be delivered to the participants via email as a secure online link, or as a hard copy booklet mailed to participants, based on participant preference. The set of questionnaires will be sent to the participants 1 week prior to their first physiotherapy session (baseline time point) and at each of the follow-up time points $(6,12$, 24 and 52 weeks). Participants who cross-over will also be sent the self-administered questionnaires at 6 and 12 weeks into the new intervention (18 and 24 weeks postrandomisation) and at 52 weeks postrandomisation. The results of these questionnaires will be marked only with the participant's identification number to ensure data confidentiality. ${ }^{58}$ The remaining outcomes will be measured by a researcher blinded to the participant's treatment allocation and include shoulder strength, scapular upward rotation angles, scapular coordinates and symptomatic onset in abduction range. These outcomes will be taken at baseline and 12 weeks postrandomisation. The outcomes to be measured in the trial are summarised in table 4. The protocol for missed physiotherapy appointments and outcome time points is outlined in the online supplementary appendix 4 .

\section{Primary outcome measures}

\section{The Melbourne Instability Shoulder Scale}

The MISS is a self-administered tool, with a total of 100 points, divided into four categories that assess pain, instability, function and occupational and sporting demands. The total score for the MISS can range between 0 and 100 points (where 0 represents no deficit and 100 the worst). The total score will be converted into as percentage of a normal healthy shoulder ${ }^{32}$ which may be more meaningful for clinical interpretation. ${ }^{61} 80$ The MISS is a valid and reliable tool, with an ability to accurately highlight the severity of a person's instability, ${ }^{59}$ and has also been shown to have a good test-retest reliability. ${ }^{32}$

\section{The Western Ontario Instability Index}

The WOSI is a self-administered tool with 21 items over the four domains of physical symptoms, sport/recreation/work, lifestyle function and emotional function. ${ }^{80}$ Each question results in a number between 0 and 100, with a total score between 0 and 2100 points (where 0 represents no deficit and 2100 the worst). The total score will be converted into a percentage of a normal healthy shoulder. ${ }^{61}{ }^{80}$ The WOSI has been shown to be responsive $^{59}$ and sensitive to change as well as being a reliable and valid tool, with a high test-retest reliability. ${ }^{61}$

\section{Data integrity}

Questionnaire data completed via the online link will be automatically scored and downloaded into a computer spreadsheet via Survey Monkey. ${ }^{81}$ Questionnaire data completed in hard copy format, once returned via mail, will be entered automatically into the computer spreadsheet by a researcher blinded to the group allocation of the participant. Data will be checked for omissions and outliers to identify potential data entry errors. Data will then be exported from the computer to SPSS software program for analyses.

\section{Blinding}

For trial validity, the participants will not be informed of which programme they are allocated to complete. ${ }^{82}$ A lack of blinding can influence participant response to treatment, compliance, use of co-interventions and risk of dropouts. ${ }^{83}$ Participants in both groups will receive the same number of treatment sessions with the same treatment duration ( 1 session of $60 \mathrm{~min}$ initial consultation and 11 sessions of 30 min follow-up consultation) and both undertake a home exercise programme. The assessor collecting the scapular and strength measures at baseline, 6 and 12 weeks will also be blinded to the 
Table 4 Outcome measures

\begin{tabular}{ll}
\hline Outcome measure & Explanation \\
\hline Primary outcomes & $\begin{array}{l}\text { Valid and reliable with good test-retest } \\
\text { The MISS }\end{array}$ \\
reliability ${ }^{32} 5960$ \\
Responsive and sensitive to change
\end{tabular}

Taken at rest, $90^{\circ}$ and EROM GHJ abduction ${ }^{62}$ Measured at $30^{\circ}, 45^{\circ}, 60^{\circ}, 90^{\circ}, 120^{\circ}, 135^{\circ}$, EROM of GHJ abduction with an inclinometer which is valid and reliable tool for measuring upward rotation ${ }^{63}$

\section{Scapular and GHJ muscle} strength

Symptomatic onset, limiting factor and angle of limiting factor in abduction

Incidence of complete glenohumeral joint dislocation

Global rating of change

Patient satisfaction score (with treatment and results)

\section{The Orebro Musculoskeletal Pain Questionnaire}

Compliance with home programme

\section{Adverse events}

Success of blinding
Assessed with a hand-held dynamometer which is a valid and reliable tool to measure shoulder strength ${ }^{64-66}$

Onset=the angle at which the participant first reports their symptoms in active abduction Limiting factor=reason for limit (p1/p2, r1/r2, apprehension)

The number of times a participant reports an episode of full glenohumeral joint dislocation (if any)

7-point Likert scale from 'completely recovered' to 'vastly worsened'. ${ }^{67} 68$ Reliable, responsive and valid 6869

5-point Likert scales from 'very satisfied' to 'very dissatisfied' $70-72$

Good reliability, validity and

responsiveness ${ }^{73} 74$

Measures psychosocial risk factors ${ }^{75} 76$

Valid and reliable tool for predicting recovery ${ }^{77} 78$

Compliance score given by trial physiotherapist from sessions 2 to 12 . The sum of scores from sessions 2 to 12 used to calculate a total compliance score at the end of the 12-week programme

Classified as minor, significant or serious (see online supplementary appendix 5)

Participants will be asked if they were aware of what programme they received.

Important to determine if protection was maintained against participant expectation effects $^{79}$

\section{Time point for assessment}

Baseline, 6, 12, 24 and 52 weeks postrandomisation*

Baseline and 12 weeks postrandomisation Baseline and 12 weeks postrandomisation

Baseline and 12 weeks postrandomisation

Baseline and 12 weeks postrandomisation

Baseline, 6, 12, 24 and 52 weeks postrandomisation*

Baseline, 6, 12, 24 and 52 weeks postrandomisation*

Baseline, 6, 12, 24 and 52 weeks postrandomisation*

Baseline, 6, 12, 24 and 52 weeks postrandomisation*

Recorded in the clinical notes from sessions 2 to 12

Recorded in the clinical notes for every session and formally assessed at 6, 12, 24 and 52 weeks postrandomisation* 12 weeks postrandomisation

A detailed explanation of secondary outcomes measures is outlined in the online supplementary appendix 5 .

${ }^{*}$ Any participant who scores less than the MCID on both primary outcomes measures after the primary 12 -week time point will be offered the alternative intervention for a subsequent 12 weeks. For these participants, outcomes measures will also be taken at 6 and 12 weeks into the new intervention (18 and 24 weeks postcross-over) as well as 52 weeks postrandomisation.

EROM, end range of motion; GHJ, glenohumeral joint; HEP, home exercise programme; MCID, minimal clinically important difference; MISS, Melbourne Instability Shoulder Score; WOSI, Western Ontario Shoulder Index.

treatment allocation of participants. Owing to the nature of the trial it is not possible to blind the treating physiotherapist to the programme they are delivering. However, the treating physiotherapists will be instructed to treat participants in both groups with the same degree of rigour, enthusiasm and optimism.

\section{Data analyses}

The primary method for data analyses with be performed using intention-to-treat principles, including post cross-over. This means that all participants will be analysed according to their randomised groups regardless of whether they crossed over or not. ${ }^{84}$ Secondary 
data analyses will be performed on a per protocol basis approach where participants will be analysed dependent on the treatment they actually receive, regardless of which treatment arm they were randomised to. ${ }^{85}$

Data analysed will focus on detecting the between-group treatment and within-group treatment effects (with effect sizes and 95\% CIs) at each of the follow-up time points. ${ }^{86}$ Primary analyses for the MISS, the WOSI, scapular coordinates, scapular angles, strength, angle of symptom onset in abduction, limiting angle in abduction, compliance with the home programme and the Orebro will be performed using linear mixed models, due to its advantages in modelling repeated measures over time. ${ }^{87}$ Mixed models will be adjusted for baseline scores. If the sample size is inappropriate for the use of a linear mixed models, ${ }^{88}$ repeated measures ANCOVA will be used and adjusted for baseline score. ${ }^{89}$

At each follow-up time point, participants will be dichotomised according to whether they achieved the MCID or not on the MISS and the WOSI. ${ }^{90} 91$ The difference between the proportions of participants who experience an improvement greater than the MCID in the two groups will then be analysed with risk ratios, risk differences and numbers needed to treat. ${ }^{83} 9293$ using intention-to-treat principles. Statistical significance will be evaluated using $\chi^{2}$ analyses. For these purposes, the MCID will be defined as above 5 points on the MISS $^{32}$ and above $10.4 \%$ on the WOSI. ${ }^{94}$

Global rating of change and satisfaction scores will be measured with Mann-Whitney U tests. ${ }^{86}$ The reason for limit in abduction and incidence of dislocation will be analysed with $\chi^{2}$ tests. $^{95}$ Adverse events, medications taken, co-interventions and success of blinding will be recorded.

\section{DISCUSSION}

In this pilot RCT, we aim to compare the Rockwood Instability programme to the Watson MDI programme for people with non-traumatic MDI. We hypothesise that the Watson programme will produce clinically and statistically better outcomes over the Rockwood programme at the primary 12-week time point, due to its focus on achieving and maintaining scapular upward rotation control and progressing exercises into functional ranges. Downward rotation of the scapula reduces joint congruency and therefore increases the potential for glenohumeral joint instability. ${ }^{196}$ People with MDI typically present with scapular downward rotation at rest ${ }^{2}$ and an exercise programme focusing on scapular upward rotation has been shown to significantly improve the strength of the scapula upward rotators and functional outcome measures of patients with MDI. ${ }^{23}$ While the Rockwood programme does involve one scapula shrug exercise in phase 2, scapular position and control is not emphasised as a primary component. As the scapulothoracic joint is the base of support from which the glenohumeral functions, ${ }^{97}$ and rehabilitation often emphasises gaining proximal control in the kinetic chain initially, a failure to address this early on may be unfavourable. In addition, the majority of the Rockwood exercises are not executed in functional ranges of motion. As strength output is position specific, ${ }^{98}$ this may be unsuitable for activity or sports-specific requirements.

We will be using several strategies to maximise and assess treatment integrity for both groups to ensure our hypothesis is tested in an unbiased manner. A comprehensive treatment manual, teacher led training of physiotherapists, quarterly workshops, the use of standardised participant information sheets (see online supplementary appendix 6) and treatment programme flow charts are methods chosen to ensure that all participants receive treatment that is standardised, accountable and reproducible. ${ }^{99} 100$ The algorithmic lay out of the treatment flow charts does permit some flexibility of exercise prescription, to ensure that treatment is relevant and specific, or at least safe and pain free for the participant.

A definitive diagnosis of MDI is challenging due to a variety of shoulder classification systems. ${ }^{2} 54$ Our definition and diagnostic criteria for MDI for this trial was developed based on convergence of validity principles. George and Delitto ${ }^{101}$ described this approach as being useful in developing classification systems where no one study or research design can provide complete validation. They defined convergence of validity for a classification system as "...evidence supporting or refuting the system (being) gathered from different sources and from the use of different methods. In the best case scenario, these sources converge and indicate similar meanings of the underlying constructs being studied" (refs. 101 and 102, p. 312). For the purpose of this trial, these principles refer to expert opinion, research on biological plausibility as well as diagnostic tests and strategies for minimising false-positive diagnosis.

In this trial, we have defined MDI as symptomatic instability in at least two directions, which is consistent with expert opinions. ${ }^{1} 245781854{ }^{103-105}$ It has also been specifically recommended ${ }^{54}$ that future studies investigating MDI should (1) consider aetiology of instability as a key element for classification ${ }^{54} 106$ (2) clearly state their inclusion criteria for MDI and whether the patient population has instability in two or three directions, ${ }^{54}$ (3) ensure that the sulcus sign produces symptomatic instability to be positive, and not just show signs of laxity ${ }^{54}$ and (4) ensure reproducible and reliable assessment between assessors for participant inclusion. ${ }^{54}$ This trial will address these criteria when making the diagnosis of MDI as outlined in this protocol.

The validity of MDI as a clinical entity is supported by research on biological plausibility. Glenohumeral joint stability is the ability to maintain centring of the humeral head at rest and through motion ${ }^{57} 107$ and depends on passive capsular restraints as well as dynamic muscular 
control. ${ }^{108}$ Studies have shown that patients with MDI have significantly larger joint capsules, ${ }^{109}$ significantly larger rotator intervals ${ }^{109} 110$ and altered muscle patterning $^{8} 105111$ when compared with normal controls. The general agreement that MDI is, in part, due to capsular redundancy, ${ }^{3-7}$ is supported by these pathoanatomical findings. On the basis of this biological plausibility, tests that evaluate the integrity of the passive restraints of the shoulder should be employed for the diagnosis of MDI. The multiple diagnostic criteria defined in this trial are commonly employed by authors investigating MDI in the literature $^{7172652104112-114}$ and support exists for individual criterion. Studies ${ }^{109} 110$ have shown that patients with MDI with a symptomatic sulcus sign had significantly longer rotator intervals on MRI compared with controls. The major static constraint against inferior instability is provided by the rotator interval complex, and its redundancy results in a sulcus. ${ }^{109} 115$ The patients in these studies had no history of trauma and no structural damage as seen on MRI. ${ }^{109}{ }^{110}$ Yoldas et al ${ }^{116}$ evaluated anterior and posterior translation testing on 48 MDI participants and compared this to evaluation under anaesthesia. There was a significantly greater degree of glenohumeral translation of the symptomatic shoulder compared with the non-symptomatic shoulder using these tests when awake as well as under anaesthesia. The anterior apprehension test and anterior draw test have also been shown to have a high diagnostic value for anterior shoulder instability when compared with radiographic or arthroscopic findings. ${ }^{113} 117$ Although these studies were investigating patients with traumatic structural instability, the presence of a positive test in the absence of a structural lesion on MRI has some validity for diagnosing atraumatic instability.

Despite the limitations in diagnosing $\mathrm{MDI}^{36}{ }^{54}$ there are aspects of our diagnostic criteria that are likely to improve the diagnostic accuracy. First, we are combining more than one type of test to assess any one direction of instability; draw tests and the apprehension tests. This is likely to increase the diagnostic accuracy for any one direction of instability. ${ }^{117}$ Second, these tests must produce the patient's symptoms, which reduces the likelihood that patients with directional laxity only will be included. Third, MRI evaluation will exclude participants with any structural damage to the shoulder. This reduces the likelihood that a participant with a traumatic unidirectional instability will be included. ${ }^{14} 35$ These methods reduce the likelihood of a false-positive diagnosis ${ }^{14} 41$ and therefore increases the validity of our approach. We also aim to keep our participant group as heterogeneous as possible by excluding participants with primary neck pathology, connective tissue disorders and volitional instability.

Based on the convergence of this quantitative and qualitative research, as well as the expertise of one of the authors (LW) comprising over 25 years of clinical practice as a shoulder physiotherapist, the diagnostic criteria used for MDI in this study have acceptable validity.

\section{CONCLUSION}

This project will establish the effect of two standardised exercise-based management programmes on the outcomes of people with non-traumatic MDI. This will establish guidelines for exercise prescription, improve the outcomes of people with MDI and lay foundations for larger RCTs for exercise of shoulder instability.

\section{Twitter Follow Sarah Warby at @warby_sarah}

Acknowledgements The authors wish to acknowledge LifeCare clinics of Victoria for providing clinic locations and forfeiting their service fees to assist the process of this trial. The authors also wish to acknowledge Shoulder and Elbow Physiotherapists Australasia (SEPA), Sports Medicine Australia (SMA) and La Trobe University for providing funding for this study.

Contributors SAW, the primary author, was involved in writing this protocol by creating drafts, responding to edits and suggestions by the coauthors and editing the final paper for submission for publication. TP, JJF, AJH, LW, SB and $R L$ were all involved in writing this protocol by editing drafts, suggesting and writing new content, editing protocol flow charts and editing protocol tables. TP, RL, SB acted as the trial assessors and were the physiotherapists who have volunteered to treat or assess participants for this trial, free of charge.

Funding This work was supported by Sports Medicine Australia (SMA), \$2000 Research Grant (no grant number) (SAW); La Trobe University Research Grants, \$1000 awarded 2013, 2014, 2015 (no grant numbers); Imaging @ Olympic Park Sports Medicine, by providing reduced cost MRIs to study participants (no grant number); LifeCare clinics of Victoria, by forfeiting service fees to study participants (no grant number); Shoulder and Elbow Physiotherapists Australasia (SEPA), \$2500 Research Grant 2016 (no grant number) (SAW).

Disclaimer These funding sources have no role in the design of this study and will not have any role during its execution, analyses, interpretation of the data, or decision to submit results.

Competing interests The Australian Postgraduate Award scheme was given to SAW's PhD scholarship. One of the authors of this paper (LW) has been the primary developer of the Watson MDI programme and teaches elements of the programme on shoulder courses; and trains trial physiotherapists. SB, SAW, TP and RL are casually employed by LW to assist with these shoulder courses. In this trial, the blinding of participants, blinding of the assessor of outcome measures, concealed allocation of treatment groups and data sharing between all investigators will ensure the Watson programme is tested against the Rockwood programme in an unbiased manner.

Ethics approval The trial has received ethical approval from La Trobe University Human Ethics Committee, Faculty of Health Sciences (FHEC12/201) and has been prospectively registered with the Australian New Zealand Clinical Trials Registry (\#ACTRN12613001240730).

Provenance and peer review Not commissioned; externally peer reviewed.

Data sharing statement All investigators will maintain full autonomy and involvement in the design, conduct and reporting of the trial with all having full access to the final data.

Open Access This is an Open Access article distributed in accordance with the Creative Commons Attribution Non Commercial (CC BY-NC 4.0) license, which permits others to distribute, remix, adapt, build upon this work noncommercially, and license their derivative works on different terms, provided the original work is properly cited and the use is non-commercial. See: http:// creativecommons.org/licenses/by-nc/4.0/

\section{REFERENCES}

1. Bahu MJ, Trentacosta N, Vorys GC, et al. Multidirectional instability: evaluation and treatment options. Clin Sports Med 2008;27:671-89. 
2. Beasley L, Faryniarz DA, Hannafin JA. Multidirectional instability of the shoulder in the female athlete. Clin Sports Med 2000;19:331-49.

3. Guerrero P, Busconi B, Deangelis N, et al. Congenital instability of the shoulder joint: assessment and treatment options. J Orthop Sports Phys Ther 2009;39:124-34

4. An YH, Friedman RJ. Multidirectional instability of the glenohumera joint. Orthop Clin North Am 2000;31:275-83.

5. Neer CS, Foster CR. Inferior capsular shift for involuntary inferior and multidirectional instability of the shoulder. A preliminary report. $J$ Bone Joint Surg Am Vol 1980;62:897-908.

6. Mallon WJ, Speer KP. Multidirectional instability: current concepts. J Shoulder Elbow Surg 1995;4(Pt 1):54-64.

7. Ide J, Maeda S, Yamaga M, et al. Shoulder-strengthening exercise with an orthosis for multidirectional shoulder instability: quantitative evaluation of rotational shoulder strength before and after the exercise program. J Shoulder Elbow Surg 2003;12:342-5.

8. Illyés Á, Kiss RM. Kinematic and muscle activity characteristics of multidirectional shoulder joint instability during elevation. Knee Surg Sports Traumatol Arthrosc 2006;14:673-85.

9. Wallmann HW. Overview of shoulder injuries, pathologies, and disorders. Home Health Care Manag Pract 2010;22:286-8.

10. Hiemstra LA, Kirkley A. Shoulder instability in female athletes. Sports Med Arthrosc 2002;10:50-7.

11. Liavaag S, Svenningsen $S$, Reikeras $\mathrm{O}$, et al. The epidemiology of shoulder dislocations in Oslo. Scand J Med Sci Sports 2011;21: e334-40.

12. Owens BD, Dawson L, Burks R, et al. Incidence of shoulder dislocation in the United States military: demographic considerations from a high-risk population. J Bone Joint Surg Am 2009;91:791-6.

13. Zarins B, Rowe C. Current concepts in the diagnosis and treatment of shoulder instability in athletes (C). Boston, MA: The American College of Sports Medicine: Massachusetts General Hospital, 1984;5:444-8 (cited 16 8005433, mg8). http://0-ovidsp.ovid.com. alpha2.latrobe.edu.au/ovidweb.cgi?T=JS\&PAGE=reference\& $\mathrm{D}=$ ovfta\&NEWS=N\&AN=00005768-198410000-00003

14. Lewis A, Kitamura T, Bayley JIL. (ii) The classification of shoulder instability: new light through old windows! Curr Orthop 2004:18:97-108.

15. Wnorowski DC. Medscape 2014 (cited 21 June 2014). http:// emedicine.medscape.com/article/1262368-overview

16. Yamaguchi K, Flatow EL. Management of multidirectional instability. Clin Sports Med 1995;14:885-902.

17. Burkhead WZ, Rockwood CA. Treatment of instability of the shoulder with an exercise program. $J$ Bone Joint Surg Am 1992;74:890-6.

18. Finnoff $J$, Doucette $S$, Hicken $G$. Glenohumeral instability and dislocation. Phys Med Rehabil Clin N Am 2004;15:v-vi, 575-605.

19. Warby SA, Pizzari T, Ford JJ, et al. The effect of exercise-based management for multidirectional instability of the glenohumeral joint: a systematic review. J Shoulder Elbow Surg 2014;23:128-42.

20. Warby SA, Pizzari T, Ford JJ, et al. Exercise-based management versus surgery for multidirectional instability of the glenohumeral joint: a systematic review. Br J Sports Med 2015;

21. Watson L, Warby SA, Balster S, et al. The treatment of multidirectional instability with an exercise program: part 1. Shoulder Elbow 2016:1-8.

22. Watson L, Warby SA, Balster S, et al. The treatment of multidirectional instability with an exercise program: part 2. Shoulder Elbow 2016:1-8.

23. Watson L. Functional and clinical changes in multidirectional instability of the shoulder after conservative rehabilitation [Professional Doctoral Thesis]. La Trobe University, Bundoora Australia, 2015.

24. Chan AW, Tetzlaff JM, Gøtzsche PC, et al. SPIRIT 2013 explanation and elaboration: guidance for protocols of clinical trials. BMJ 2013;346:e7586.

25. Schulz KF, Altman DG, Moher D. CONSORT 2010: updated guidelines for reporting parallel group randomised trials. Ann Intern Med 2010;152:1-8.

26. Kiss J, Damrel D, Mackie A, et al. Non-operative treatment of multidirectional shoulder instability. Int Orthop 2001;24:354-7.

27. Freedman B. Equipoise and the ethics of clinical research. $N$ Engl J Med 1987;317:141-5.

28. Mills EJ, Chan AW, Wu P, et al. Design, analysis, and presentation of crossover trials. Trials 2009;10:27.

29. Moritani TMA, deVries HAPD. Neural factors versus hypertrophy in the time course of muscle strength gain. Am J Phys Med 1979;58:115-30.
30. Rutherford OM, Jones DA. The role of learning and coordination in strength training. Europ J Appl Physiol 1986;55:100-5.

31. Rampello A, Franceschini M, Piepoli M, et al. Effect of aerobic training on walking capacity and maximal exercise tolerance in patients with multiple sclerosis: a randomised crossover controlled study. Phys Ther 2007;87:545-55.

32. Watson L, Story I, Dalziel R, et al. A new clinical outcome measure of glenohumeral joint instability: the MISS questionnaire. $J$ Shoulder Elbow Surg 2005;14:22-30.

33. Carlin J, Doyle L. Statistics for clinicians. J Paediatr Child Health 2002;38:300-4.

34. Kuroda S, Sumiyoshi T, Moriishi J, et al. The natural course of atraumatic shoulder instability. J Shoulder Elbow Surg 2001;10:100-4.

35. Gerber $\mathrm{C}$, Nyffeler R. Classification of glenohumeral joint instability. Clin Orthop Relat Res 2002;400:65-76.

36. Kuhn JE, Helmer TT, Dunn WR, et al. Development and reliability testing of the frequency, etiology, direction, and severity (FEDS) system for classifying glenohumeral instability. $J$ Shoulder Elbow Surg 2011;20:548-56.

37. Bahk M, Keyurapan E, Tasaki A, et al. Laxity testing of the shoulder: a review. Am J Sports Med 2007;35:131-44.

38. Tzannes A, Paxinos A, Callanan M, et al. An assessment of the interexaminer reliability of tests for shoulder instability. J Shoulder Elbow Surg 2004;13:18-23.

39. McFarland EG. Examination of the shoulder. New York, NY: Thieme Medical Publishers, Inc., 2006. http://books.google.com.au/ books?id=BMVAAGMRRAUC\&pg=PA204\&lpg=PA204\&dq=The +Shoulder.+New+York:+Churchill+Livingstone;+1988:165-291\& source=bl\&ots=lg6UTt318W\&sig=mDe0P7hE_-tPtZ4opkjM8VV $x S B M \& h l=e n \& s a=X \& e i=w-R t U a 7 m M e q z i Q e K w Y A w \& v e d=$ 0CC8Q6AEwAA-v=onepage\& $q=$ The $\% 20$ Shoulder.\%20New\% 20 York\%3A\%20Churchill\%20Livingstone\%3B\%201988\% 3A165-291\& $\mathrm{f}=$ false

40. Levy AS, Lintner S, Kenter K, et al. Intra- and interobserver reproducibility of the shoulder laxity examination. Am J Sports Med 1999;27:460-3.

41. Gerber C, Ganz R. Clinical assessment of instability of the shoulder. With special reference to anterior and posterior drawer tests. J Bone Joint Surg Br 1984;66:551-6.

42. Rowe C, Leffert RD. The shoulder. New York: Churchhill Livingstone, 1988:165-291.

43. Rowe CR, Zarins B. Recurrent transient subluxation of the shoulder. J Bone Joint Surg Am 1981;63:863-72.

44. McFarland EG. Examination of the shoulder: the complete guide. New York, NY: Thieme, 2006.

45. Kessel L. Clinical disorders of the shoulder. Edinburgh: Churchill Livingstone, 1982.

46. Rabin A, Irrgang JJ, Fitzgerald GK, et al. The intertester reliability of the Scapular Assistance Test. J Orthop Sports Phys Ther 2006;36:653-60.

47. Seitz AL, McClure PW, Lynch SS, et al. Effects of scapular dyskinesis and scapular assistance test on subacromial space during static arm elevation. J Shoulder Elbow Surg 2012;21:631-40.

48. Beighton P, Horan F. Orthopedic aspects of the Ehlers-Danlos syndrome. J Bone Joint Surg Br Vol 1969;51:444-53.

49. Boyle KL, Witt $P$, Riegger-Krugh $C$. Intrarater and interrater reliability of the Beighton and Horan Joint Mobility Index. $J$ Ath Train 2003;38:281-5.

50. Tong HC, Haig AJ, Yamakawa K. The Spurling test and cervical radiculopathy. Spine 2001;27:156-9.

51. Shah CK, Rajshekhar V. Reliability of diagnosis of soft cervical disc prolapse using Spurling's test. Br J Neurosurg 2004;18:480-3

52. Kiss RM, Illyés Á, Kiss J. Physiotherapy vs. capsular shift and physiotherapy in multidirectional shoulder joint instability. J Electromyogr Kinesiol 2010;20:489-501.

53. Altchek DW, Warren RF, Skyhar MJ, et al. T-plasty modification of the Bankart procedure for multidirectional instability of the anterior and inferior types. J Bone Joint Surg Am 1991;73:105-12.

54. McFarland EG, Kim TK, Park HB, et al. The effect of variation in definition on the diagnosis of multidirectional instability of the shoulder. J Bone Joint Surg Am 2003;85A:2138-44.

55. Main CJ, Sowden G, Hill JC, et al. Integrating physical and psychological approaches to treatment in low back pain: the development and content of the STarT Back trial's 'high-risk' intervention (StarT Back; ISRCTN 37113406). Physiotherapy 2012:98:110-16.

56. Boudreau SA, Farina D, Falla D. The role of motor learning and neuroplasticity in designing rehabilitation approaches for musculoskeletal pain disorders. Man Ther 2010;15:410-14. 
57. Kraemer WJ, Adams K, Cafarelli E, et al., American College of Sports Medicine. American College of Sports Medicine position stand. Progression models in resistance training for healthy adults. Med Sci Sports Exerc 2002;34:364-80.

58. Quinn MJ. Confidentiality. In: Elliott P, Cuzick J, English D, Stern R eds. Geographical and environmental epidemiology: methods for small area studies. Oxford Scholarship Online: Oxford University Press, 1996:1-9.

59. Plancher KD, Lipnick SL. Analysis of evidence-based medicine for shoulder instability. Arthroscopy 2009;25:897-908.

60. Kirkley A, Griffin S, McLintock H, et al. The development and evaluation of a disease-specific quality of life measurement tool for shoulder instability: the Western Ontario Shoulder Instability Index (WOSI). Am J Sports Med 1998;26:764-72.

61. Salomonsson B, Ahlstrom S, Dalen N, et al. The Western Ontario Shoulder Instability Index (WOSI): validity, reliability, and responsiveness retested with a Swedish translation. Acta Orthop 2009;80:233-8.

62. Watson LA, Pizzari T, Balster S. Thoracic outlet syndrome part 2: conservative management of thoracic outlet. Man Ther 2010;15:305-14.

63. Watson L. Measurement of scapula upward rotation: a reliable clinical procedure. Br J Sports Med 2005;39:599-603.

64. Burnham RS, Bell G, Olenik L, et al. Shoulder abduction strength measurement in football players: reliability and validity of two field tests. Clin J Sport Med 1995;5:90-4.

65. Leggin BG, Neuman RM, lannotti JP, et al. Intrarater and interrater reliability of three isometric dynamometers in assessing shoulder strength. J Shoulder Elbow Surg 1996;5:18-24.

66. Hayes K, Walton JR, Szomor ZL, et al. Reliability of 3 methods for assessing shoulder strength. J Shoulder Elbow Surg 2002;11:33-9.

67. Beurskens AJHM, de Vet HCW, Köke AJA. Responsiveness of functional status in low back pain: a comparison of different instruments. Pain 1996;65:71-6.

68. Kamper S. Global rating of change scales. Aust J Physiother 2009;55:289.

69. Kamper S, Maher C, Mackay G. Global rating of change scales: a review of strengths and weaknesses and considerations for design. J Man Manip Ther 2009;17:163-70.

70. Bombardier CMD. Outcome assessments in the evaluation of treatment of spinal disorders: summary and general recommendations. Spine 2000;25:3100-3.

71. Deyo R, Battie M, Beurskens AJH, et al. Outcome measures for low back pain research: a proposal for standardized use. Spine 1998;23:2003-13.

72. Hudak PL, Wright JG. The characteristics of patient satisfaction measures. Spine 2000;25:3167-77.

73. Ferrer M, Pellise $\mathrm{F}$, Escudero $\mathrm{O}$, et al. Validation of a minimum outcome core set in the evaluation of patients with back pain. Spine 1976;31:1372-9.

74. Mannion A, Elfering A, Staerkle R, et al. Outcome assessment in low back pain: how low can you go? Eur Spine $J$ 2005;14:1014-26.

75. Linton SJ, Boersma K. Early identification of patients at risk of developing a persistent back problem: the predictive validity of the Orebro Musculoskeletal Pain Questionnaire. Clin J Pain 2003;19:263-9.

76. Linton SJPD, Hallden KBA. Can we screen for problematic back pain? A screening questionnaire for predicting outcome in acute and subacute back pain. Clin J Pain 1998;14:209-15.

77. Maher C, Grotle M. Evaluation of the predictive validity of the Orebro musculoskeletal pain screening questionnaire. Clin J Pain 2009;25:666-70.

78. Westman A, Linton SJ, Ohrvik J, et al. Do psychosocial factors predict disability and health at a 3-year follow-up for patients with non-acute musculoskeletal pain? A validation of the Orebro Musculoskeletal Pain Screening Questionnaire. Eur J Pain 2008;12:641-9.

79. Fergusson D, Glass KC, Waring D, et al. Turning a blind eye: the success of blinding reported in a random sample of randomised, placebo controlled trials. BMJ 2004;328:432.

80. Kirkley $A$, Werstine $R$, Ratjek $A$, et al. Prospective randomised clinical trial comparing the effectiveness of immediate arthroscopic stabilization versus immobilization and rehabilitation in first traumatic anterior dislocations of the shoulder: long-term evaluation. Arthroscopy 2005;21:55-63.

81. Finley R, Finley C. Survey Monkey 2014. https://www. surveymonkey.com

82. Bridgman S, Dainty K, Kirkley A, et al. Practical aspects of randomisation and blinding in randomised clinical trials. Arthroscopy 2003;19:1000-6.
83. Moher D, Sally H, Kenneth FS, et al. CONSORT 2010 Explanation and Elaboration: updated guidelines for reporting parallel group randomised trials. BMJ 2010;340:c869.

84. Laude EA, Duffy NC, Baveystock $C$, et al. The effect of helium and oxygen on exercise performance in chronic obstructive pulmonary disease: a randomised crossover trial. Am J Respir Crit Care Med 2006;173:865-70.

85. Morden JP, Lambert PC, Latimer N, et al. Assessing methods for dealing with treatment switching in randomised controlled trials: a simulation study. BMC Med Res Methodol 2011;11:4.

86. Portney LG, Watkins MP. Foundations of clinical research. In: Cohern M, ed. 3rd edn. Upper Saddle River, NJ: Julie Levin Aexander, 2009.

87. Krueger C, Tian L. A comparison of the general linear mixed model and repeated measures ANOVA using a dataset with multiple missing data points. Biol Res Nurs 2004;6:151-7.

88. Bolker BM, Brooks ME, Clark CJ, et al. Generalized linear mixed models: a practical guide for ecology and evolution. Trends Ecol Evol 2009;24:127-35.

89. Van Breukelen GJ. ANCOVA versus change from baseline: more power in randomised studies, more bias in nonrandomised studies. J Clin Epidemiol 2006;59:920-5.

90. Dworkin RH, Turk DC, McDermott MP, et al. Interpreting the clinical importance of group differences in chronic pain clinical trials: IMMPACT recommendations. Pain 2009;146:238-44.

91. Snapinn S, Jiang Q. Responder analyses and the assessment of a clinically relevant treatment effect. Trials 2007;8:31.

92. Busse JW, Guyatt GH. Optimizing the use of patient data to improve outcomes for patients: narcotics for chronic noncancer pain. Expert Rev Pharmacoecon Outcomes Res 2009;9 171-9.

93. Smith C, Grimmer-Somers K. The treatment effect of exercise programmes for chronic low back pain. J Eval Clin Pract 2010;16:484-91.

94. Kirkley A, Griffin S, McLintock H, et al. The development and evaluation of a disease-specific quality of life measurement tool for shoulder instability. The Western Ontario Shoulder Instability Index (WOSI). Am J Sports Med 1998;26:764-72.

95. Campbell M, Swinscow T. Statistics at square one. (11th edition). Singapore: Wiley-Blackwell. 2009:87-153.

96. Kikuchi K, Itoi E, Yamamoto N, et al. Scapular inclination and glenohumeral joint stability: a cadaveric study. J Orthop Sci 2008;13:72-7.

97. Levangie PK, Norkin C. Joint structure and function: a comprehensive analyses. 3rd edn. Canada: MacLennan \& Petty Pty Limited, 2001.

98. Moriello C, Mayo NE. Development of a position-specific index of muscle strength to be used in stroke evaluation. Arch Phys Med Rehabil 2006;87:1490-5.

99. Borrelli B, Sepinwall D, Ernst D, et al. A new tool to assess treatment fidelity and evaluation of treatment fidelity across 10 years of health behavior research. J Consult Clin Psychol 2005;73:852-60.

100. Perepletchikova F, Treat TA, Kazdin AE. Treatment integrity in psychotherapy research: analysis of the studies and examination of the associated factors. J Consult Clin Psychol 2007;75: 829-41.

101. George SZ, Delitto A. Clinical examination variables discriminate among treatment-based classification groups: a study of construct validity in patients with acute low back pain. Phys Ther 2005;85:306-14

102. Ford JJ, Surkitt LD, Hahne AJ. A classification and treatment protocol for lowback disorders. Part 2-directional preference management for reducible discogenic pain. Phys Ther Rev 2011;16:423-237.

103. Barden JM, Balyk R, Raso VJ, et al. Atypical shoulder muscle activation in multidirectional instability. Clin Neurophysiol 2005;116:1846-57.

104. Tillander B, Lysholm M, Norlin R. Multidirectional hyperlaxity of the shoulder: results of treatment. / Hyperlaxite pluridirectionnelle de I ' epaule: resultats du traitement. Scand J Med Sci Sports 1998;8:421-5.

105. Morris AD, Kemp GJ, Frostick SP. Shoulder electromyography in multidirectional instability. J Shoulder Elbow Surg 2004;13: 24-9.

106. Rockwood C, Thomas S, Matsen F. Subluxations and dislocations about the glenohumeral joint. In: Rockwood CA, Green DP, Bucholz RW, eds. Fractures in adults. 3rd edn. Philadelphia JB Lippincott, 1991:1164-1208.

107. Darlow B. Neuromuscular retraining for multidirectional instability of the shoulder-a case study. N Z J Physiother 2006;34:60-5. 
108. Hayes K, Callanan M, Walton J, et al. Shoulder instability: management and, rehabilitation. J Orthop Sports Phys Ther 2002;32:497-509.

109. Lee HJ, Kim NR, Moon SG, et al. Multidirectional instability of the shoulder: rotator interval dimension and capsular laxity evaluation using MR arthrography. Skeletal Radiol 2013;42:231-8

110. Hsu YC, Pan RY, Shih YY, et al. Superior-capsular elongation and its significance in atraumatic posteroinferior multidirectional shoulder instability in magnetic resonance arthrography. Acta Radiol 2010;51:302-8.

111. Kronberg M, Brostrom LA, Nemeth G. Differences in shoulder muscle activity between patients with generalized joint laxity and normal controls. Clin Orthop 1989;269:181-92.

112. Joseph TA, Williams JS, Brems JJ. Laser capsulorrhaphy for multidirectional instability of the shoulder: an outcomes study and proposed classification system. Am J Sports Med 2003;31:26-35.
113. van Kampen DA, van den Berg $\mathrm{T}$, van der Woude $\mathrm{HJ}$, et al Diagnostic value of patient characteristics, history, and six clinical tests for traumatic anterior shoulder instability. J Shoulder Elbow Surg 2013;22:1310-19.

114. Misamore GW, Sallay PI, Didelot W. A longitudinal study of patients with multidirectional instability of the shoulder with sevento ten-year follow-up. J Shoulder Elbow Surg 2005;14: 466-70.

115. Jost B, Koch PP, Gerber C. Anatomy and functional aspects of the rotator interval. J Shoulder Elbow Surg 2000;9:336-41.

116. Yoldas EA, Faber KJ, Hawkins RJ. Translation of the glenohumeral joint in patients with multidirectional and posterior instability: awake examination versus examination under anesthesia. J Shoulder Elbow Surg 2001;10:416-20.

117. Farber AJ, Castillo R, Clough $\mathrm{M}$, et al. Clinical assessment of three common tests for traumatic anterior shoulder instability. J Bone Joint Surg Am 2006;88:1467-74. 